\title{
POEMS Syndrome: A Case Report of a patient with CIDP
}

Mansour $\mathbf{M}^{*}$, Souissi W, Beyrouti R and Mrissa R

Department of Neurology, Military Hospital of Tunis, Tunisia

*Corresponding author: Mansour M, Department of Neurology, Military Hospital of Tunis, Tunisia, Tel: 21671561 804; E-mail: Mansour.malek18@yahoo.com

Rec: Feb 06, 2017, Acc: Mar 06, 2017, Pub: Mar 12, 2017

Copyright: @ 2017 Mansour M. This is an open-access article distributed under the terms of the Creative Commons Attribution License, which permits unrestricted use, distribution, and reproduction in any medium, provided the original author and source are credited.

\begin{abstract}
POEMS syndrome is a multisystem disorder associated with polyneuropathy, organomegaly, endocrinopathy, a monoclonal protein (M-protein), and skin changes. Polyneuropathy is the most common initial symptom and it can hide other features of this disorder. We describe a patient with chronic inflammatory demyelinating polyneuropathy (CIDP) associated with extra-neurologic signs fulfilling the diagnosis criteria of POEMS syndrome to emphasize the importance of meticulous physical examination leading to challenging diagnosis.
\end{abstract}

Keywords: Cerebral Palsy; 1960's; CTG monitoring; Intra-uterine hypoxia; False conclusions; Misdirected court action; ACOG - AAP guidelines; Future action

\section{Case Report}

A 67-year-old man, with a previous history of lumbar plasmocytoma diagnosed in 2004 and successfully treated with high radiation dose, presented in July 2016 with distal lower limbs paresthesia and weakness progressing proximally and to the upper limbs. Within 9 months he was unable to walk or stand. He also reported erectile dysfunction without any sphincter problems.

Clinical examination revealed symmetric limbs weakness more pronounced distally and in the lower limbs with generalized hyporeflexia, impairment of touch, pain, and temperature sensation in both hands, a severe affection of position and vibration senses in the higher limbs and an ataxic gait with a positive Romberg's sign. Examination of the cranial nerves was normal. At the general physical examination, we noticed a bilateral pretibial edema, hypertrichosis and multiples skin angioma (Figure 1) [1].

Neurophysiological testing showed a severe demyelinating polyneuropathy with significant secondary axonal loss fulfilling the EFNS/ PNS diagnostic criteria for CIDP. The cerebrospinal fluid protein level was of $0.91 \mathrm{~g} / \mathrm{l}$ with 9 leucocytes.

Full blood count, renal and hepatic function tests, glycaemia, autoantibody screen, cryoglobulin screen, vitamin B12 and folate levels and thyroid function tests were within normal ranges. We excluded infections of lyme, hepatitis and human immunodeficiency virus via serologic tests.

Magnetic resonance imaging (MRI) of spine showed discrete lesions of low signal intensity in both T1 and T2-weighted images of a lumbar vertebra. Biopsy of this lesion demonstrated a plasmacytoma.

Serum electrophoresis revealed an IgG-lambda monoclonal serum protein. There was no Bence-Jones proteinuria. Bone marrow biopsy showed normal cellularity and did not detect any bone marrow plasma cell involvement, which excluded multiple myeloma.

Abdominal ultrasound showed a mildly enlarged spleen but no hepatomegaly. Heart ultrasound showed pulmonary hypertension.
Serum testosterone was reduced to $6.8 \mathrm{nmol} / 1$ 9reference interval 9-33 $\mathrm{nmol} / \mathrm{l}$ ) with a high prolactin level of $340 \mathrm{mU} / \mathrm{l}$ (reference interval 31.5-399 mU/l). Vascular endothelial growth factor (VEGF) levels couldn't be evaluated because of its non-availability in our country. A diagnosis of POEMS syndrome was made referring to POEMS syndrome criteria. Treatment with radiotherapy was limited by his high-risk of toxicity. Initial improvement in strength was noted within 2 weeks following high-dose of oral steroids. Four months after the diagnosis, he was able to walk unaided.

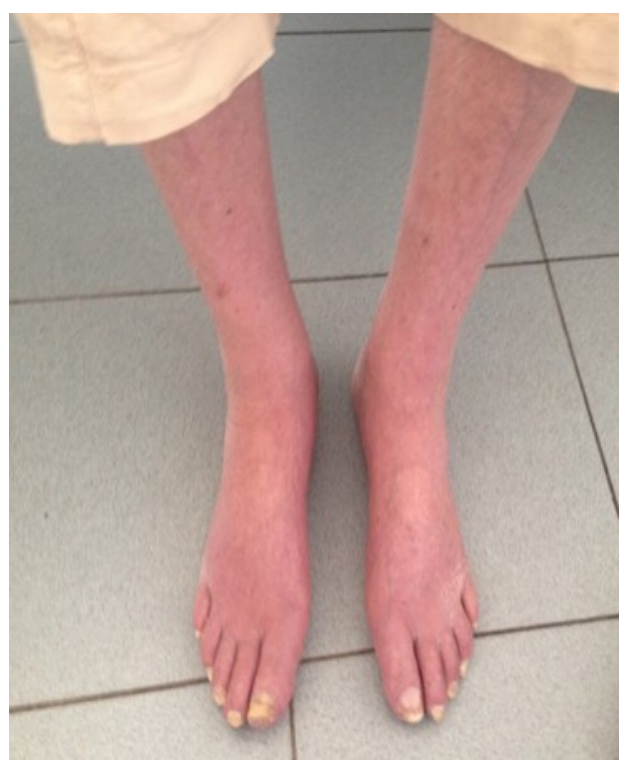

Figure 1: Bilateral pretibial edema, hypertrichosis and multiples skin angioma.

\section{Discussion}

Chronic inflammatory demyelinating polyneuropathy (CIDP) typically presents as a progressive or relapsing, symmetric sensorimotor neuropathy with proximal and distal muscle weaknesses developing over two or more months. The condition is often associated 
Page 2 of 2

with an elevated cerebrospinal fluid protein level and demyelinating nerve-conduction studies [2]. Our patient presented a typical form of CIDP. Before making the diagnosis of an idiopathic CIDP, a report of associated pathology should be made. This includes infections (Lyme, $\mathrm{B}$ hepatitis and human immunodeficiency virus), diabetes, dysthyroid, autoimmune diseases, Vitamin B12 deficiency and drug abuse or paraneoplasic neuropathy. Detecting extra neurologic signs in a patient presenting with CIDP can be the key for the diagnosis. Some features should draw the clinician's attention and lead to the diagnosis of POEMS syndrome. The most common abnormalities are skin changes especially hyperpigmentation and hypertrichosis that can be either generalized or limited to a body part, organomegaly such as hepatomegaly, splenomegaly and lymphadenopathy and some biological abnormalities especially endocrine abnormalities such as hypogonadism, hypothyroidism and diabetes, and an evidence of a monoclonal plasmaproliferative disorder [3].

Pulmonary hypertension found in our patient is unusual feature in POEMS syndrome but it was reported. Pleural effusion, ascites, and/or cardiac effusion still the first symptoms to appear in more than half of patients with POEMS syndrome and it revealed the disease in one series in $12 \%$ of patients and coincides with neuropathy in $14 \%$ [4].

POEMS syndrome is revealed by neuropathy in $50 \%$ of cases and almost $60 \%$ of those patients were initially diagnosed as having idiopathic CIDP. Before development of typical systemic manifestations, POEMS neuropathy should be distinguished from others CIDP by her clinical profile. Her onset can be either insidious, as our patient, or rapidly progressing. It is symmetrical and ascending. Clinically, it involves both motor and sensory nerves, begins distally, and has a progressive proximal spread. Patients often describe dysesthesia followed by a progressive weakness that overshadows the sensory impairment. Associated cranial or autonomic system involvement is rare $[5,6]$.

To retain the diagnosis, most authors agree that patients with POEMS syndrome should have 3 or more of the 5 features: polyneuropathy, organomegaly, endocrinopathy, a monoclonal protein (M-protein), and skin changes. Some others have proposed that the presence of 2 major criteria, including a monoclonal plasmaproliferative disorder and polyneuropathy, in addition to the existence of 1 minor criterion, is sufficient for diagnosis. The suggested minor criteria include sclerotic bone lesions, organomegaly, edema, endocrinopathy, papilledema, and skin changes. Our patient filled the diagnosis criteria of POEMS. In fact, he had polyneurothy, a monoclonal plasma proliferative protein, skin changes, endocrinopathy and sclerotic bone lesion.

POEMS syndrome is often undiagnosed or misdiagnosed especially when its symptoms mimic other disorders as cancers or metastases. It has a rapidly progressive course; therefore, early diagnosis is important.
His pathogenesis is poorly understood. The production of various inflammatory cytokines, such as interleukins (IL-1 and IL-6) and VEGF, by abnormal plasma cells has probably a direct causal role in various symptoms of the condition [7].

Treatment is not standardized but most authors agree that for an isolated plasmacytoma, which was the case of our patient, radiation is preferred. When this treatment is not allowed, many strategies have been used including plasmapheresis, intravenous immunoglobulin, azathioprine and corticosteroids. For those patients with diffuse disease, systemic therapy is indicated [8]. Clinical responses to prednisone occurred in approximately $22 \%$ to $56 \%$ of patients. Usulally, systemic and skin symptoms respond sooner than do symptoms of neuropathy. The target of this case is to emphasize that the general examination of patients with CIDP is extremely important before retaining an idiopathic CIDP.

\section{Conclusion}

POEMS syndrome is not rare. The neuropathy stills a common and an initial feature of the disease. Its clinical and electrical profile should be well known by the clinicians but sometimes its diagnosis requires a combination of biological and especially examination findings in patient with CIDP. Recognition of this disorder leads to early diagnosis and proper treatment.

\section{References}

1. Chee CE, Dispenzieri A, Gertz MA (2010) Amyloidosis and POEMS syndrome. Expert Opin Pharmacother 11: 1501-1514.

2. Dispenzieri A (2014) POEMS syndrome: 2014 update on diagnosis, riskstratification and management. Am J Hematol 89: 214-223.

3. Del Río Prado AF, Reza Albarrán AA, Gómez Pérez FJ (2015) Male aged 31 years with polyneuropathy, prostration and hypogonadism. Gac Med Mex. 151: 256-259.

4. Nakanishi T, Sobue I, Toyokura Y, Nishitani H, Kuroiwa Y, et al. (1984) The Crow-Fukase syndrome: A study of 102 cases in Japan. Neurology. 34: 712-720.

5. Nasu S, Misawa S, Sekiguchi Y, Shibuya K, Kanai K, et al. (2012) Different neurological and physiological profiles in POEMS syndrome and chronic inflammatory demyelinating polyneuropathy. J Neurol Neurosurg Psychiatry. 83: 476-479.

6. Li J, Zhou DB (2013) New advances in the diagnosis and treatment of POEMS syndrome. Br J Haematol. 161: 303-315.

7. Kastritis E, Terpos E, Anagnostopoulos A, Xilouri I, Dimopoulos MA (2011) Angiogenetic factors and biochemical markers of bone metabolism in POEMS syndrome treated with high-dose therapy and autologous stem cell support. Clin Lymphoma Myeloma. 7: 73-76.

8. Cai QQ, Wang C, Cao XX, Cai H, Zhou DB, et al. (2015) Efficacy and safety of low-dose lenalidomide plus dexamethasone in patients with relapsed or refractory POEMS syndrome. Eur J Haematol. 95: 325-330. 